\title{
Phase Equilibria in a System of "Breathing" Molecules
}

\author{
Jianzhong $\mathrm{Wu}^{*}$ and John Prausnitz ${ }^{\dagger}$
}

Department of Chemical Engineering, University of California, Berkeley, and Chemical

Sciences Division, Lawrence Berkeley National Laboratory, Berkeley, CA 94720

Keywords: breathing molecules, solid-fluid equilibrium, statistical mechanics

\begin{abstract}
It is now well known that details in the intermolecular potential can significantly affect the qualitative features of a phase diagram where temperature is plotted against density for the coexistence curves among fluid and solid phases. While previous calculations of phase diagrams have assumed a time-invariant potential function, this report concerns the phase diagram for "breathing" molecules, i.e., molecules whose strength of intermolecular attraction fluctuates in time. Such fluctuations can occur in biomacromolecules where an active site can switch between "on" and "off" positions. Phase-equilibrium calculations were performed for molecules that have a periodic (breathing) attractive force in addition to the conventional intermolecular forces. The phase diagram for such molecules is as expected when the "breathing" properties are independent of density. However, when (more realistically), the "breathing" properties are density dependent, the phase diagram exhibits dramatic changes. These calculations may be useful for interpreting experimental data for protein precipitation, for plaque formation in blood vessels and for scaffold-supported tissue formation.
\end{abstract}

\footnotetext{
* Current address: Department of Chemical Engineering, University of California, Riverside, CA 92521

${ }^{\dagger}$ To whom correspondence should be addressed 


\section{Introduction}

For mixtures of fluids, phase-equilibrium calculations in science and engineering are based on a variety of models for representing thermodynamic properties; these models vary from those based on relatively rigorous statistical mechanics to those derived from molecular-thermodynamic or phenomenological considerations and finally to those that are strictly empirical [1]. Common to essentially all of these models is the implicit assumption that for compact (spherical or globular) molecules, the vibrational state of the molecules has no appreciable effect on intermolecular forces and that, therefore, phaseequilibrium calculations for fluids containing such molecules need not be concerned with molecular vibration.

On the other hand, many years of research in biochemistry have shown that the active sites of a globular protein are often inside a pocket that vibrates with a regular frequency such that, when the pocket is open, it is able to interact strongly (binding) with another molecule whereas, when the pocket is closed, such strong interaction is not possible due to steric hindrance [2]. Here "open" and "closed" are relative terms that describe intermolecular binding; "closed" does not necessarily mean association is totally prohibited nor does "open” necessarily mean association is complete.

We report here some initial studies toward calculation of phase equilibrium for a one-component fluid where the molecules may or may not interact strongly depending on whether or not the molecules are "open" or "closed". We designate such molecules "breathing" molecules. We expect that calculations reported here may find application for some biological systems where condensation depends, at least in part, on the vibrational state, that is, on the fraction of molecules that, at a given time, are "open" for strong interactions. 


\section{A Model for Breathing Molecules}

To fix ideas, we consider first the interaction potential between two spherical molecules as shown in Figure 1. Regardless of whether or not the molecules are open or closed, the potential is of the van der Waals form reflecting hard sphere repulsion and attractive dispersion forces. However, if both molecules are open, the potential also includes a specific strong interaction.

In Figure 1, the diameter of the molecule is $\sigma$ and the depth of the conventional (i.e., van der Waals) potential is $\varepsilon$. The range of attraction is reflected in the exponent $n$; the range falls as $n$ rises.

When both molecules are open, there is an additional association potential characterized by $\varepsilon_{A}$ which is assumed to be appreciably larger than $\varepsilon$. For open molecules, the specific association force is "on" when the center-to-center distance between two molecules is less than $r_{c}$, and when the angles $\theta_{1}$ and $\theta_{2}$ are equal to or less than a critical angle $\theta_{c}$. In this work, we set $r_{c}=1.05 \sigma$, and $\theta_{c}=27^{\circ}$, as used in SAFT theory for associated fluids [3]. The reasonable values of these parameters will not affect the qualitative features of our calculations. The bonding probability, designated by $\phi$, describes the fraction of time in a vibration period that a molecule is open. For a system containing a large number of molecules, $\phi$ is the same as the fraction of "open" molecules at any moment. In general, the factor $\phi$ may depend on temperature and density. In this initial study, we assume that $\phi$ depends only on density and can vary from zero to $\phi^{0}$, the maximum probability of opening. For our preliminary purposes, we consider three simple cases:

1. Breathing independent of density, $\phi=\phi^{0}$ (Autonomous breathing)

2. Breathing declines with rising density $\phi=\phi^{0}\left(1-\rho / \rho_{0}\right)$ (Restricted breathing) 
3. Breathing increases with rising density $\phi=\phi^{0} \rho / \rho_{0} \quad$ (Cooperative breathing)

Here $\rho$ stands for the molecular density and $\rho_{0}$ corresponds to that at close packing.

For a face-centered cubic (FCC) structure, $\rho_{0} \sigma^{3}=\sqrt{2}$.

In autonomous breathing, the fraction of "open" molecules is independent of density. In restricted breathing, where the close presence of other molecules discourages breathing, the fraction of molecules in the open position declines with density, and in cooperative (synergetic) breathing, where the close presence of other molecules encourages breathing, the fraction of molecules in the open position rises with density. For simplicity, here we assume a linear dependence on density but this simplifying assumption is not essential.

In the subsequent illustrations, we consider fluid-fluid and fluid-solid equilibrium of breathing molecules. We set $\varepsilon_{A} / k T_{0}=16$ where $k$ is Boltzmann's constant and reference temperature $T_{0}$ is $298.15 \mathrm{~K}$. It is now well known that for large globular molecules, the (reduced) range of van der Waals attractive forces is smaller that that for simple (argon-like) molecules [4]. In our calculations, we use $n=6.5$.

Because we are concerned with globular biomacromolecules (e.g., proteins) in solution, our potential corresponds to a potential of mean force; the biomacromolecules are in a solvent that is here considered to be a continuous medium. Our calculations, therefore, are in the McMillan-Mayer framework.

\section{Helmholtz Energy of the Fluid Phase}

To reveal the qualitative features of the phase diagrams for breathing molecules, we use the random-phase approximation (RPA) for the Helmholtz energy. RPA is essentially a first-order perturbation where the reference system is a hard-sphere fluid and the perturbation has two parts; one part that applies to all molecules, arising from attractive van der Waals forces, and a second part that applies only to open molecules, 
arising from specific two-body attraction. The second perturbation is based on SAFT theory [3].

The fluid-phase Helmholtz energy $F$ is

$$
\begin{gathered}
\frac{F}{N k T}=\ln \left(\rho \Lambda^{3}\right)-1+x_{1} \ln x_{1}+x_{2} \ln x_{2}+\frac{4 \eta-3 \eta^{2}}{(1-\eta)^{2}}-\frac{12 \eta \varepsilon}{(n-3) k T} \\
+x_{2}[\ln X-X / 2+1 / 2]
\end{gathered}
$$

where $N$ is the total number of molecules (before association), $\Lambda$ is the thermal wavelength, $x_{1}$ is the mole fraction of "closed" molecules, $x_{2}$ is the mole fraction of "open" molecules, $\eta=\pi \rho \sigma^{3} / 6$ is the packing fraction, and $X$ is the SAFT parameter, representing the fraction of unbonded sites. Because we distinguish between "open" and "closed" molecules, the Helmholtz energy contains a contribution from the (ideal) entropy of mixing "open" and "closed" molecules.

\section{Helmholtz Energy of the Solid Phase}

The solid phase is assumed to have a face-centered-cubic structure, as shown in Figure 2. To represent the Helmholtz energy of the solid phase, we use the free-volume cell theory where the free volume per molecule $v_{0}$ is approximated by [4]

$$
v_{0}=\frac{8}{\sqrt{2}}(a-\sigma)^{3} .
$$

Here $a$ is the center-to-center distance between two nearest-neighbor molecules; density $\rho$ is related to $a$ by $\rho / \rho_{0}=(\sigma / a)^{3}$. Substitution then gives

$$
v_{0}=\frac{8}{\sqrt{2}} \sigma^{3}\left[\left(\rho_{0} / \rho\right)^{1 / 3}-1\right] .
$$

In Equation (3) the coefficient $8 / \sqrt{2}$ is obtained from a refined free-volume theory of Velasco et al. where $v_{0}$ was obtained from a size scaling of a Wigner-Seitz cell [5]. Compared with molecular-simulation results for hard spheres, this modified free-volume theory provides a Helmholtz energy of the solid phase that is much better than that 
obtained from the "classical" free-volume theory [where the coefficient in Eq.(3) is $1 / \sqrt{2}]$

As for the fluid phase, the Helmholtz energy of the solid includes a contribution from the reference hard-sphere crystal, a perturbation due to van der Waals attraction, and a contribution from intermolecular bonding:

$$
\frac{F}{N k T}=-\ln \left(v_{0} / \Lambda^{3}\right)+x_{1} \ln x_{1}+x_{2} \ln x_{2}-\frac{z_{1} \varepsilon}{2 k T}\left(\frac{\rho}{\rho_{c}}\right)^{n / 3}+x_{2}[\ln X-X / 2+1 / 2] .
$$

In Eq.(4), $z_{1}=12$ is the number of nearest neighbors in a FCC crystal. The mixing terms on the right-hand side of Eq.(4) take into account that the crystalline phase contain both "open" and "close" molecules. The van der Waals attraction includes only nearestneighbor interactions. Here, we extended the SAFT equation for fluids to solids by replacing the contact value of the radial distribution function in a hard-sphere fluid by that in a hard-sphere solid. To obtain the contact value of the radial distribution function for the hard-sphere solid, we apply the compressibility equation

$$
Z^{h s}=1+4 \eta g^{h s}(\sigma)
$$

where the compressibility factor for the hard-sphere solid, $Z^{h s}$, is calculated selfconsistently from the cell model.

\section{Phase-Equilibrium Calculations}

For each phase, chemical potential $\mu$ is obtained from

$$
\mu=\left(\frac{\partial F}{\partial N}\right)_{T, V}
$$

where $V$ is the total volume, and pressure is obtained from

$$
P=-\left(\frac{\partial F}{\partial N}\right)_{T, N}
$$

A fluid-fluid coexistence curve is obtained from the relations 


$$
\begin{aligned}
& \mu^{\alpha}=\mu^{\beta} \\
& P^{\alpha}=P^{\beta}
\end{aligned}
$$

where $\alpha$ and $\beta$ designate two separate phases. For a fluid, the Helmholtz energy to calculate pressure and chemical potential is given by Equation (1). At a fixed temperature, we solve for equilibrium densities $\rho^{\alpha}$ and $\rho^{\beta}$. If no solution is found, the temperature is above the critical temperature for fluid-fluid equilibrium; there is only one fluid phase.

For fluid-solid equilibrium, we also use Equations (8) and (9) but now, for phase $\alpha$ we use Equation (1) for the liquid-phase Helmholtz energy while for phase $\beta$ we use Equation (4) for the solid-phase Helmholtz energy. Again, we search for densities $\rho^{\alpha}$ and $\rho^{\beta}$ that satisfy both equations of phase equilibrium, i.e., Eqs.(8) and (9).

\section{Results}

Figure 3 gives phase diagrams for two limiting cases: one has no association and in the other, all molecules can associate. In both limiting cases, the fluid-fluid binodal line is below the freezing line, as in typical aqueous protein solutions. These two phase diagrams are similar but, as expected, the fluid-fluid critical temperature for the associating fluid is higher than that for the non-associated fluid because, when all molecules are open, the attractive forces are larger.

Figure 4 shows phase diagrams for cooperative breathing and for restricted breathing. Again, the two diagrams are similar in shape but the fluid-fluid critical temperature for cooperative breathing is larger than that for restricted breathing. Figure 4 indicates that the breathing pattern of molecules could have a significant effect on the phase diagram.

Figure 5 shows the effect of $\phi^{0}$, the maximum fraction of open molecules, on fluid-fluid critical temperature $T_{c}$ and on critical density $\rho_{c}$. For all $\phi^{0}$, compared with 
the autonomous breathing where the probability of bonding is independent of density, $T_{c}$ is larger for cooperative breathing and smaller for restrictive breathing. Similar trends apply for $\rho_{c}$. Surprisingly, for molecules with restricted breathing, the critical temperature falls with increasing probability of association, and becomes zero when $\phi^{0}$ is larger than around 0.62 . The absence of a vapor-liquid critical temperature for fluids with both van der Waals attraction and association has not been observed before.

Figure 6 presents the effect of van der Waals attraction on the critical temperature and density of breathing molecules. Whereas $\varepsilon / k T_{0}$ was set equal to 2 in Figures 3,4 and 5, in Figure 6, $\varepsilon / k T_{0}$ varies from zero to 10 ; in all cases, however, $\varepsilon_{A} / k T_{0}=16$.

The calculations shown in Figure 6 indicate that when the conventional van der Waals attraction is weak ( $\varepsilon / k T_{0}$ less than about 3.1 ), there is no fluid-fluid equilibrium. In other words, restricted breathing suppresses the fluid-fluid equilibrium. This result appears consistent with the long-known result that in a model for a binary liquid mixture of components $\mathrm{D}$ and $\mathrm{C}$, liquid-liquid phase separation cannot occur when postulating a chemical equilibrium of the form $\mathrm{C}+\mathrm{D} \rightarrow \mathrm{CD}$ unless physical attractive forces are also included in the model. Without physical forces, chemical solvation models assume that the "true" species (D, C, CD) form an ideal solution and an ideal solution is stable at all compositions [6].

Finally, Figure 7 shows phase diagrams for autonomous, cooperative and restricted breathing. For all cases, as $\phi^{0}$ rises, the phase diagram shifts toward higher reduced temperatures $k T / \varepsilon$. Relative to autonomous breathing, the width of the fluidfluid region is larger when the fraction of open molecules $\phi$ depends on density, especially for cooperative breathing. 
Perhaps most interesting is the effect of breathing on the crystallization temperature. Suppose that $\phi^{0}=0.9$ and that we have a protein solution at advanced temperature whose concentration $\rho \sigma^{3}$ is 0.2 . We now cool the mixture. What is the reduced crystallization temperature? For autonomous breathing it is 0.54 , for cooperative breathing, 0.65 and for restricted breathing, 0.25. Although restricted breathing introduces additional attraction between molecules, the critical temperature for this case is significantly lower than that without additional attraction. In other words, restricted breathing inhibits a fluid-fluid phase transition.

\section{Conclusion}

Some preliminary calculations have been made to determine the effect of molecular breathing on the phase diagrams of protein solutions. Here "breathing" refers to the effect of molecular vibrations that allow us to distinguish between "open" and "closed" molecules; two "closed" molecules or one "closed" and one "open" molecule interact according to conventional intermolecular forces while two "open" molecules (in addition to conventional forces) interact strongly through association. Our thermodynamic description corresponds to a two-component model in the McMillanMayer framework where the solvent is not a component but a background medium. We considered three cases: first, where the fraction of "open" molecules is independent of density, second, where it declines, and third where it increases linearly with density. Calculated results show that for the three cases, the liquid-liquid connodal line and the freezing line differ and, more important, differ appreciably from the case where breathing is neglected.

Numerous refinements are possible. Our purpose here is only to introduce the concept of "breathing" molecules and to suggest that phase-equilibrium calculations for such molecules may be useful for interpreting condensation (phase-change) phenomena 
for biomacromolecules as observed, for example, in growth of human tissue, in clogging of arteries or in formation of biofilms in sewage pipes or barnacles on ships [7].

\section{Lists of Symbols}

$a: \quad$ nearest center-to-center distance between molecules in the solid phase

$F: \quad$ Helmholtz energy

$g(\sigma)$ : contact value of radial distribution function

$k: \quad$ Boltzmann's constant

$N: \quad$ total number of molecules (before association)

$n: \quad$ exponent parameter in the potential funciton

$P: \quad$ pressure

$r: \quad$ center-to-center distance between molecules

$r_{c}: \quad$ critical association distance

$T: \quad$ temperature

$T_{0}: \quad$ reference temperature, $298.15 \mathrm{~K}$

$V: \quad$ volume

$v_{0}: \quad$ free volume in the cell model

$X: \quad$ SAFT parameter

$x_{1}: \quad$ mole fraction of "closed" molecules

$x_{2}: \quad$ mole fraction of "open" molecules

$Z: \quad$ compressibility factor

$z_{1}: \quad$ number of nearest neighbors

$\varepsilon: \quad$ van der Waals attraction parameter

$\varepsilon_{A}: \quad$ association energy 
$\eta=\pi \rho \sigma^{3} / 6$ : packing fraction

$\theta_{1}, \theta_{2}:$ orientation angles

$\theta_{c}: \quad$ critical bonding angle

$\Lambda: \quad$ thermal wavelength

$\mu: \quad$ chemical potential

$\rho: \quad$ molecular density

$\rho_{0}: \quad$ close-packed density

$\sigma: \quad$ molecular diameter

$\phi: \quad$ molecular vibration parameter

$\phi^{0}: \quad$ maximum probability of bonding

\section{Acknowledgments}

For financial support, the authors are grateful to the Office for Basic Sciences, US Department of Energy and to the National Science Foundation. The authors are also grateful to David Schafer and Adam P. Arkin for insightful discussions on possible biorelevance of breathing molecules.

\section{Reference:}

[1] J.V. Sengers .et al. (Eds.), Equations of State for Fluids and Fluid Mixtures, Elsevier Science, New York, 2000.

[2] E. K. Wilson, Chemical \& Engineering News, 78 (20000), 42-45.

[3] W.G. Chapman et al, Ind. Eng. Chem. Res., 29 (1990), 1709-1721.

[4] P. G. Debenedetti, Metastable Liquids, Princeton University Press, 1996.

[5] E. Velasco, L. Mederos and G. Navascues, Langmuir, 14 (1998) 5652-5655.

[6] I. Prigogine and R. Defay, Chemical Thermodynamics, Longmans, 1954.

[7] G. O'Toole, H.B. Kaplan and R. Kolter, Annual Review of Microbiology, 54 (2000) 49-79. 
Figure Captions

Figure 1 Pair potential $u=u_{0}+u_{A}$ between two breathing molecules includes a van der Waals attraction $\left(u_{0}\right)$ and an association between breathing sites $\left(u_{A}\right)$.

Figure 2 Free volume for each molecule in the solid face according to a cell model.

Figure 3 Effect of bonding on the phase diagram: (a) no association,(b) all molecules can associate. Here $\varepsilon / k T_{0}=2$.

Figure 4 Phase Diagrams of Cooperative and restricted breathing molecules (for $\phi^{0}=1$ and $\left.\varepsilon / k T_{0}=2\right)$.

Figure 5 Effect of breathing pattern on the critical point of liquid-liquid equilibrium (for $\left.\varepsilon / k T_{0}=2\right)$.

Figure 6 Effect of van der Waals interaction on the critical point of liquid-liquid equilibrium (for restricted and cooperative breathing, $\phi^{0}=1$ ).

Figure 7 Effect of breathing on phase diagrams: (a) autonomous breathing, (b) cooperative breathing, (c) restricted breathing. Here $\varepsilon / k T_{0}=2$. 
Figure 1

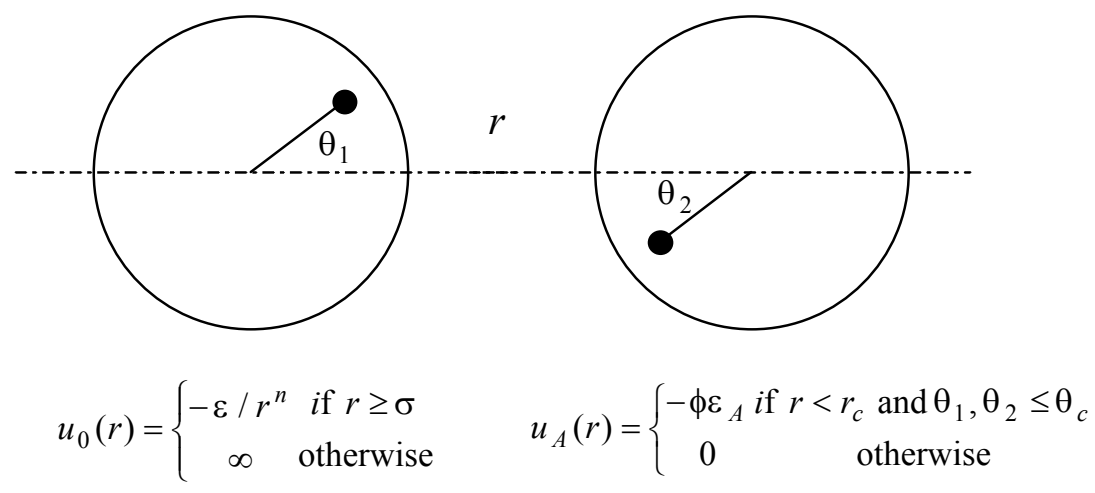


Figure 2

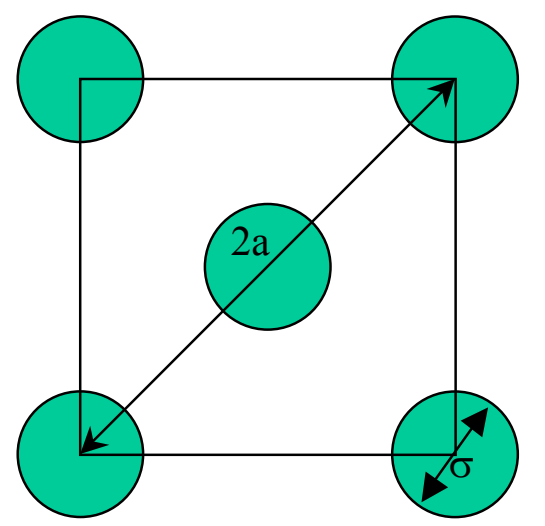

$$
\begin{aligned}
v_{0} / \sigma^{3} & \approx 8(a / \sigma-1)^{3} / \sqrt{2} \\
& =8\left[\left(\rho_{0} / \rho\right)^{1 / 3}-1\right]^{3} / \sqrt{2}
\end{aligned}
$$

$v_{0}$ : free volume of each molecule

$$
\rho_{0} \sigma^{3}=\sqrt{2}
$$


Figure 3

a) no association

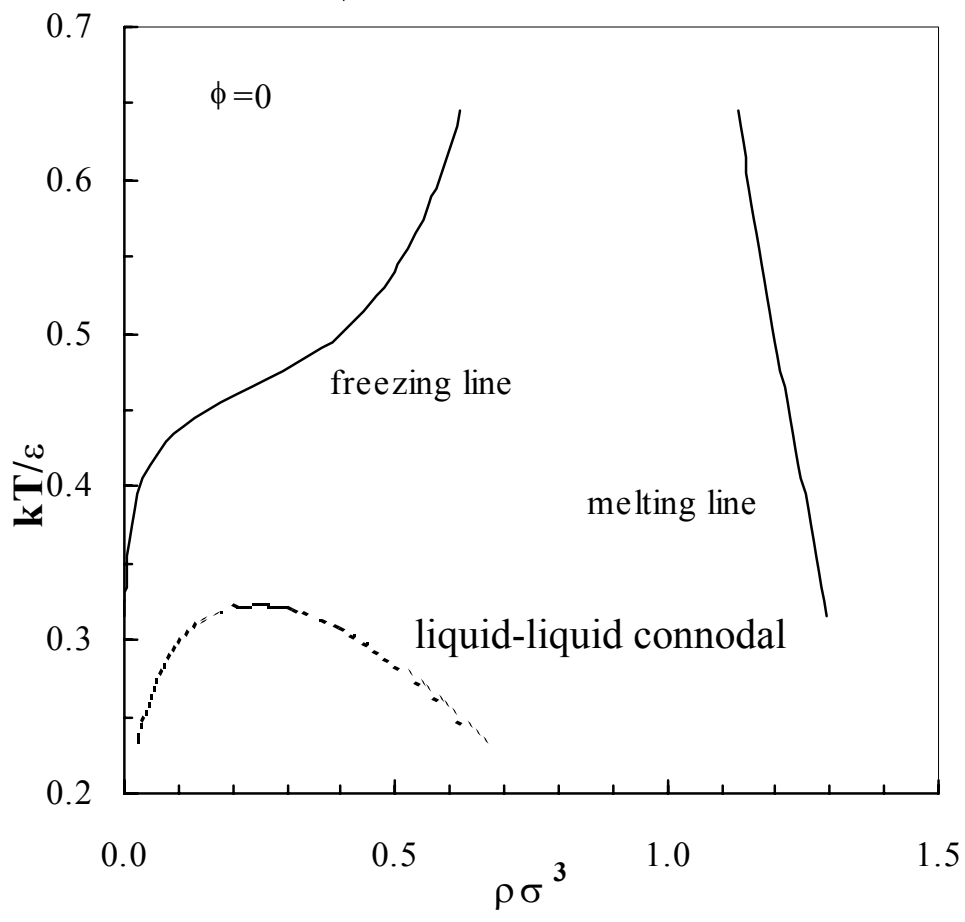

b) all molecules can associate

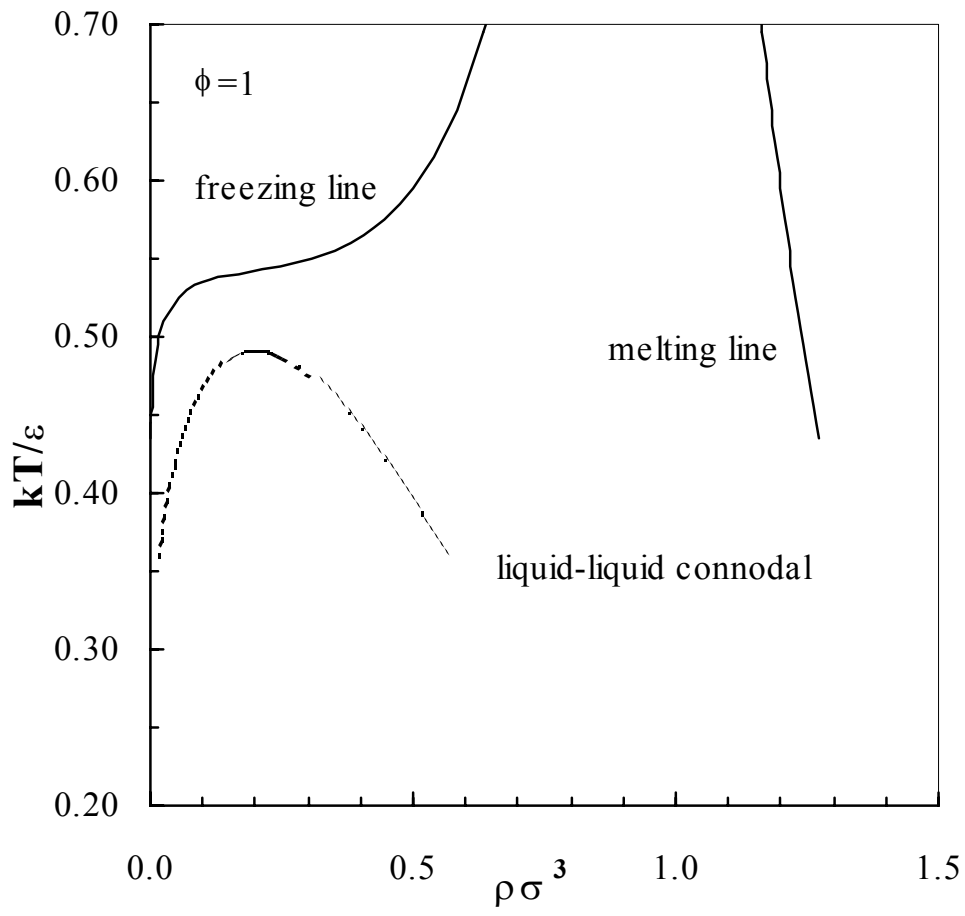


Figure 4
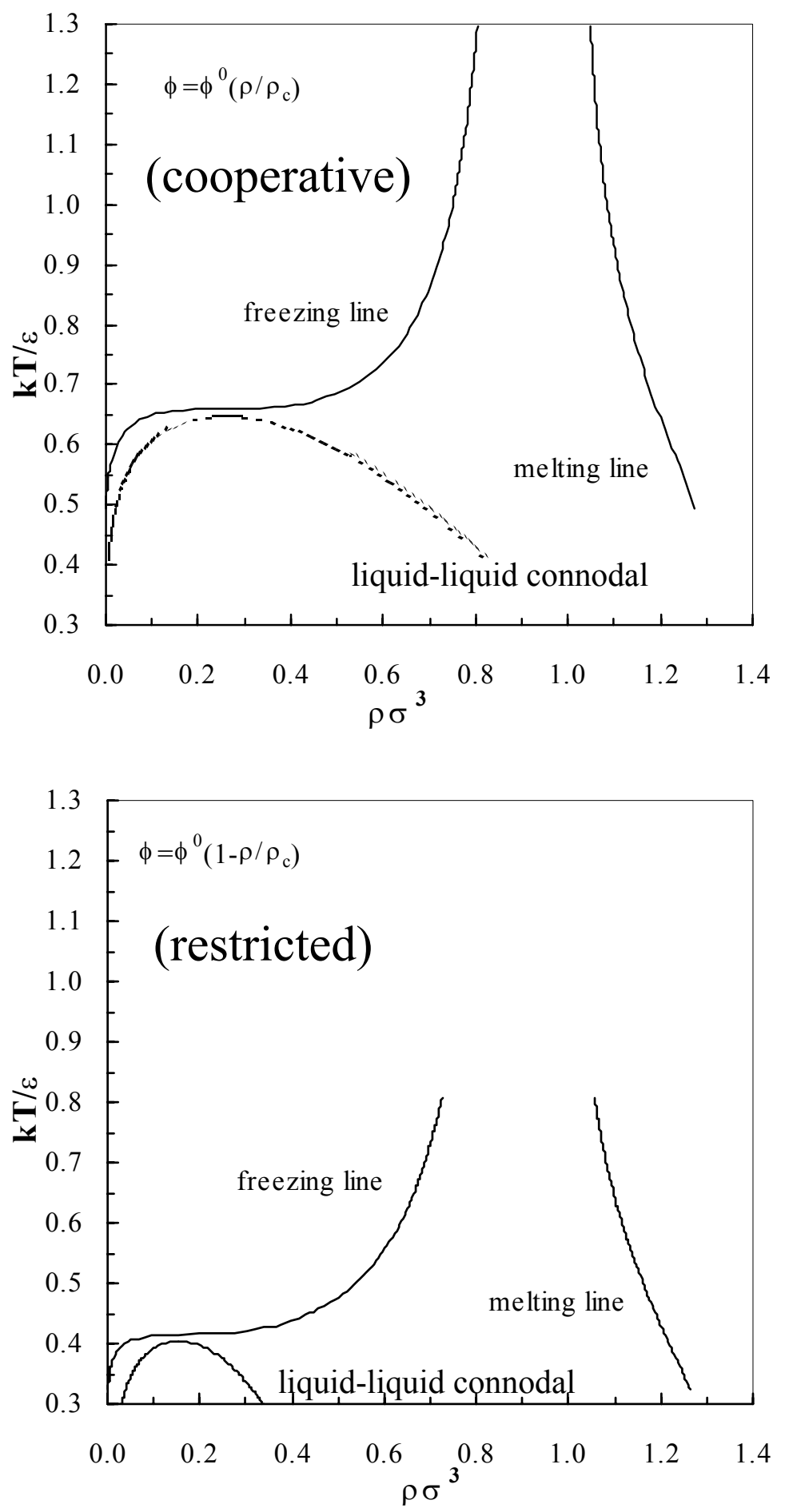
Figure 5
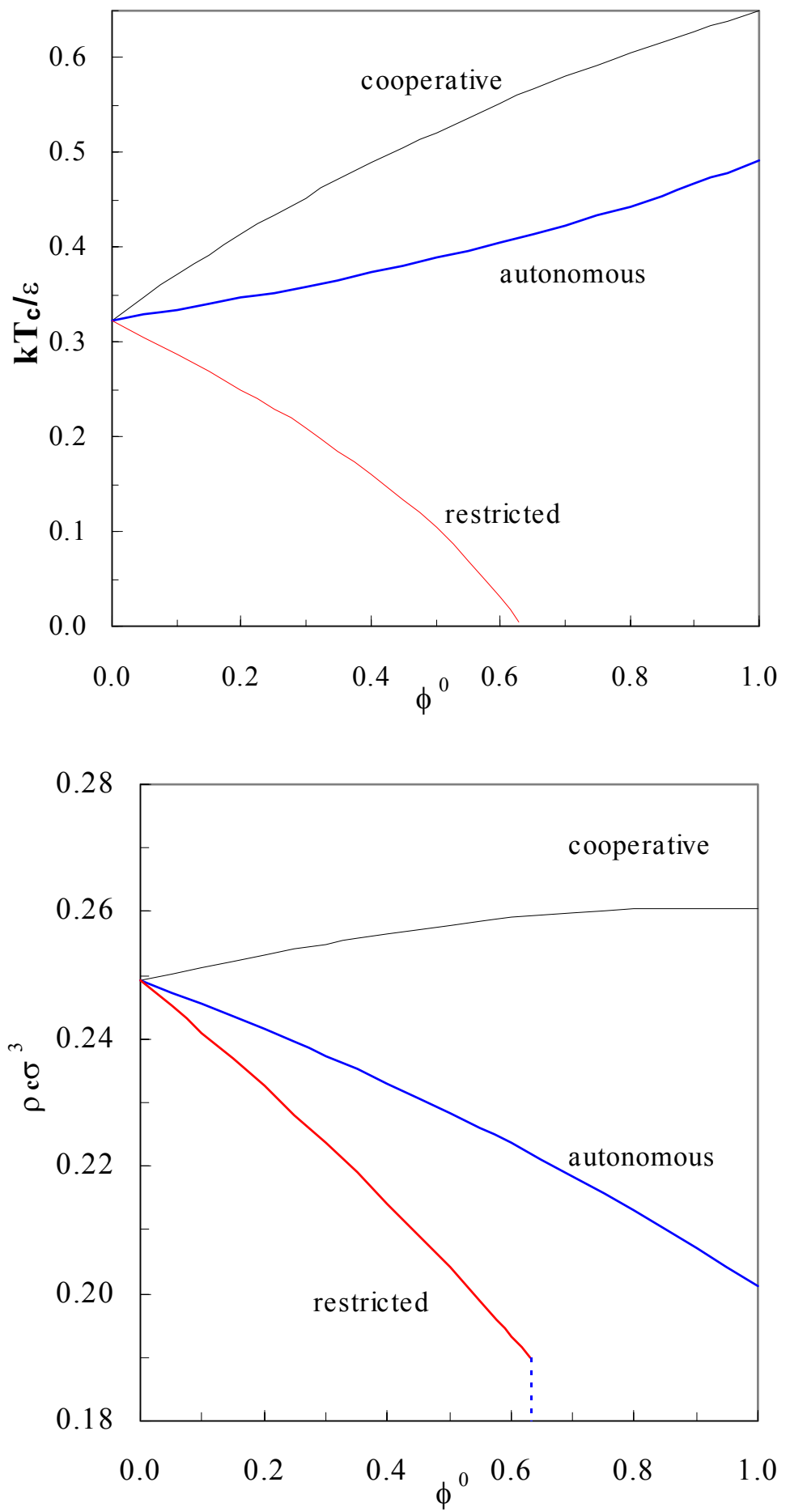
Figure 6
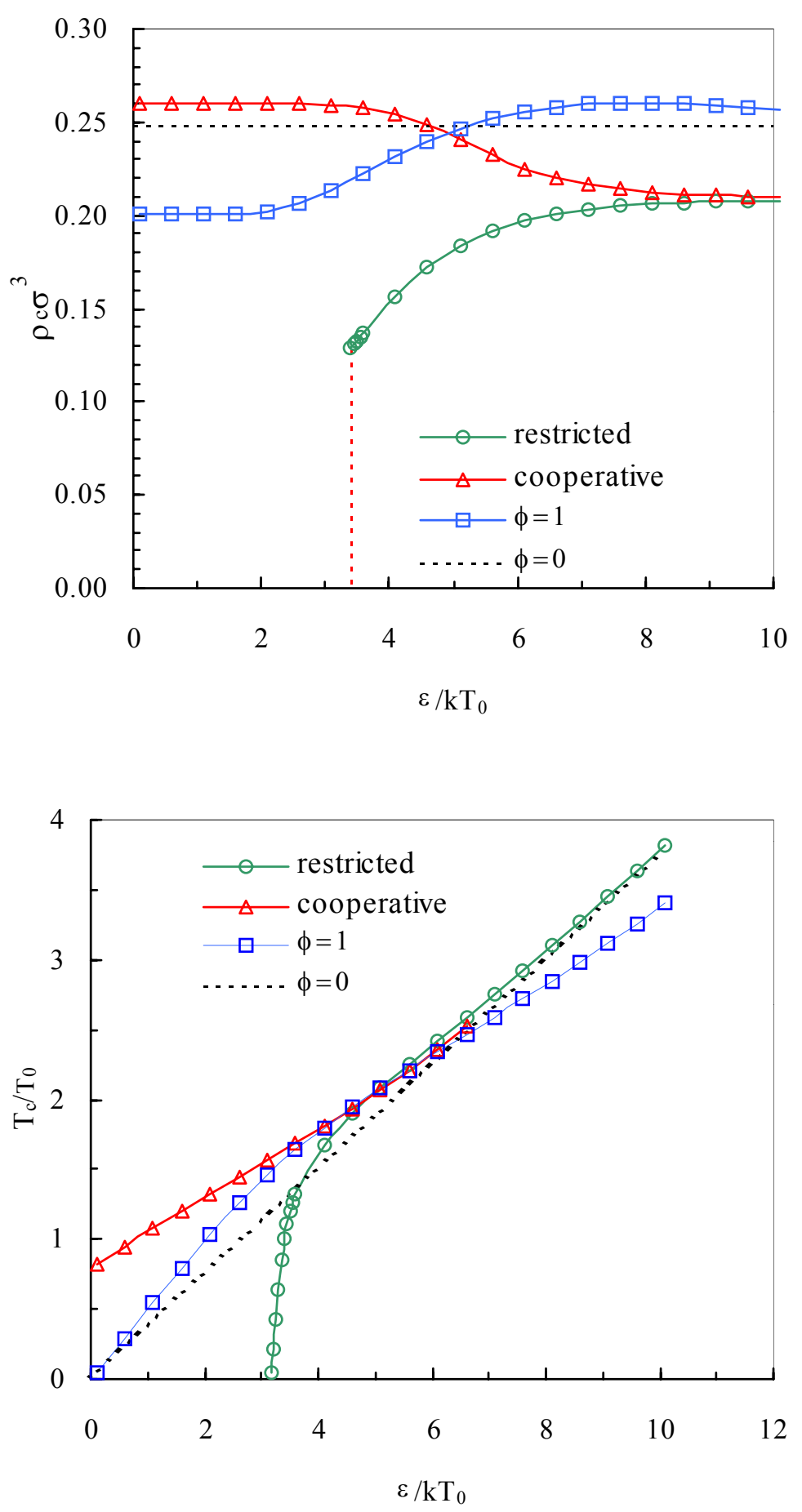
Figure 7
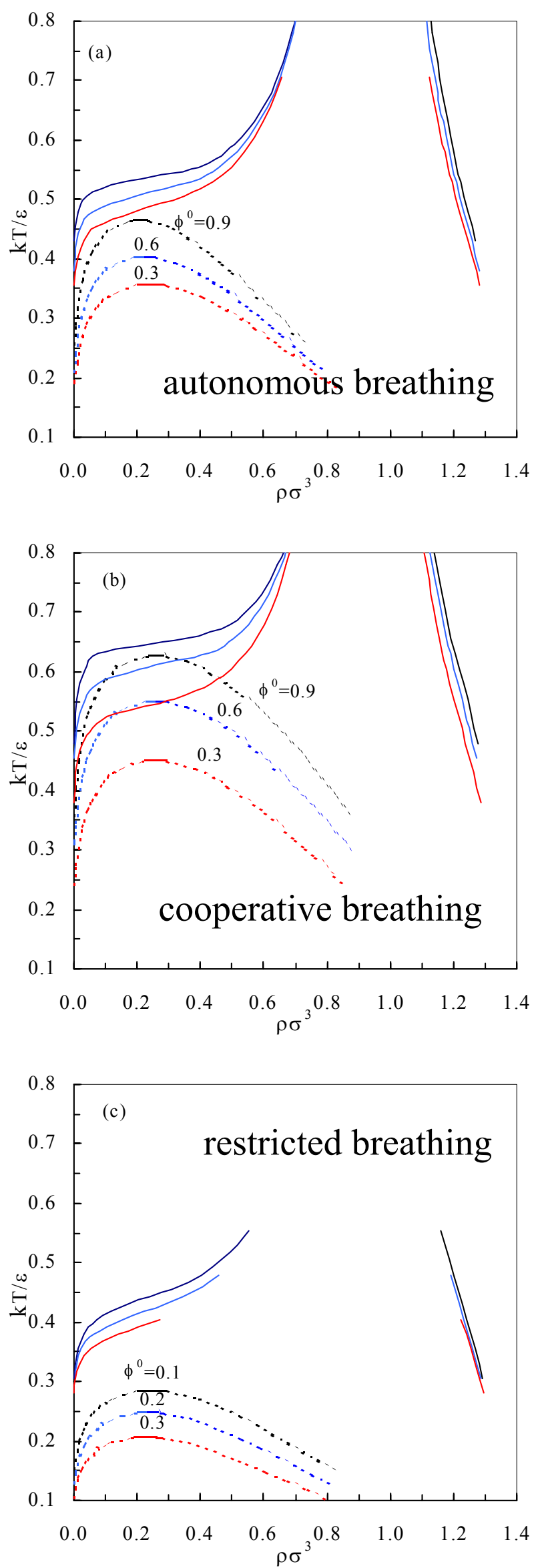